\title{
Cytological and architectural heterogeneity in ductal carcinoma in situ of the breast
}

\author{
Cecily M Quinn, Julian L Ostrowski
}

\begin{abstract}
Aim-The traditional architecture based classification system of ductal carcinoma in situ (DCIS) has been criticised on the grounds that individual lesions often show more than one pattern resulting in a large mixed category. New DCIS classification systems have emphasised the importance of cytological grade, which is reputed to be more uniformly expressed throughout a lesion. This study investigates the hypothesis that cytological heterogeneity is less common than architectural heterogeneity within DCIS lesions.
\end{abstract}

Methods-121 cases of DCIS were graded as poorly, intermediately, or well differentiated according to a recently developed classification system that employs cytonuclear morphology as the major diagnostic criterion. Cases were categorised as pure when only one grade was present and as mixed if more than one grade was observed. Architecturally the cases were classified as solid, cribriform, micropapillary, or papillary and were described as pure if only one architectural pattern was present and as mixed if more than one pattern was seen. The incidence of cytological heterogeneity was compared with that of architectural heterogeneity. The presence of necrosis was assessed as an independent parameter and the relation to DCIS grade evaluated.

Results-Using the cytology based classification system 102 cases $(84 \%)$ were classified as pure ( 65 poorly differentiated, 25 intermediately differentiated, and 12 well differentiated) and 19 cases (16\%) as mixed. Extensive necrosis was observed in $61(50 \%)$ cases and was closely correlated to DCIS grade. Architecturally 46 cases $(38 \%)$ were classified as pure (38 solid, 5 cribriform, 2 micropapillary, and 1 papillary) and $75(62 \%)$ as mixed.

Conclusions-Cytological heterogeneity is much less common than architectural heterogeneity in DCIS lesions. The assessment of cytonuclear morphology is therefore likely to provide more consistent information about DCIS, particularly in small biopsy specimens where only part of the lesion may be available for examination.

(F Clin Pathol 1997;50:596-599)

Keywords: ductal carcinoma in situ; cytological grade; architectural patterns
Ductal carcinoma in situ (DCIS), once an uncommon disease, now accounts for up to $13 \%$ of all newly diagnosed breast cancers. This largely results from the detection of DCIS by mammography screening. With the increased numbers of cases available for study it has become apparent that DCIS is a heterogeneous condition with differing clinical outcomes, ${ }^{1-11}$ emphasising the need for a prognostically relevant histological classification system. The traditional system classifies DCIS according to architectural patterns and the presence or absence of luminal necrosis. ${ }^{12}$ Comedo, solid, cribriform, micropapillary, and papillary forms are described. The development of this system has greatly facilitated the recognition of DCIS and its distinction from epitheliosis. This system is disadvantaged by the fact that many cases show more than one architectural pattern resulting in a large mixed category. ${ }^{114}$ Apart from comedo DCIS, which appears to be a more aggressive lesion, architectural patterns are not predictive of biological behaviour. In the past few years a number of alternative classification systems have been proposed. ${ }^{4}{ }^{11} 14-17$ In general these have emphasised the importance of cytonuclear features, which appear to be more closely related to clinical outcome and biological characteristics. Proponents of the new classification systems also suggest that cytonuclear morphology is more constant throughout a lesion than architectural pattern. This is of obvious importance as decisions regarding patient management are currently based on the assessment of biopsy specimens where the entire lesion may not be available for examination. Using the system described by Holland et $a l^{15}$ that employs cytonuclear morphology as the key diagnostic criterion we graded 121 cases of DCIS into one or more categories and compared the degree of heterogeneity with that observed when the cases were classified using the traditional architecture based system.

\section{Materials and methods}

The study population comprised 121 patients with DCIS, 26 of whom also had early invasive carcinoma (microinvasion or invasion up to $5 \mathrm{~mm}$ ). All patients were diagnosed and treated at the General Infirmary at Leeds between 1984 and 1994. Cases were identified using the SNOMED Diagnostic Retrieval System. Age at presentation ranged from 29 to 88 years (median 55). Sixty six patients (55\%) were symptomatic and in $55(45 \%)$ DCIS was detected mammographically. Seventy nine patients $(65 \%)$ underwent mastectomy and 42

Correspondence to: 
(35\%) had local excision. All histological sections from each case were reviewed in detail.

DCIS was graded as poorly, intermediately, or well differentiated according to the method

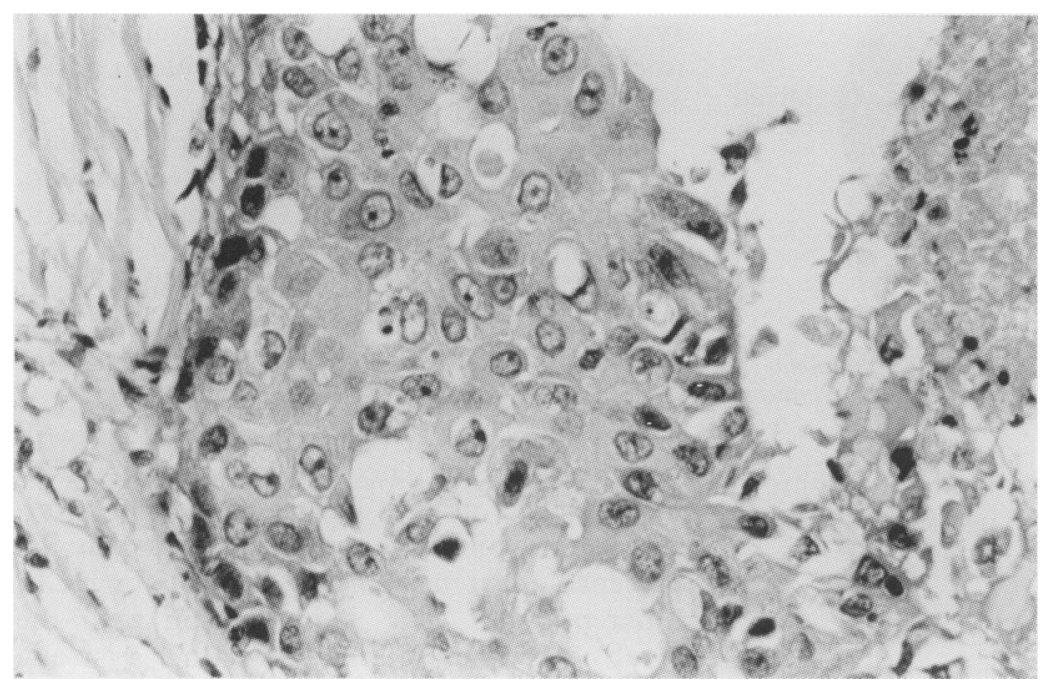

Figure 1 Poorly differentiated DCIS showing a solid growth pattern and luminal necrosis The nuclei are markedly pleomorphic and there is no cell polarisation.

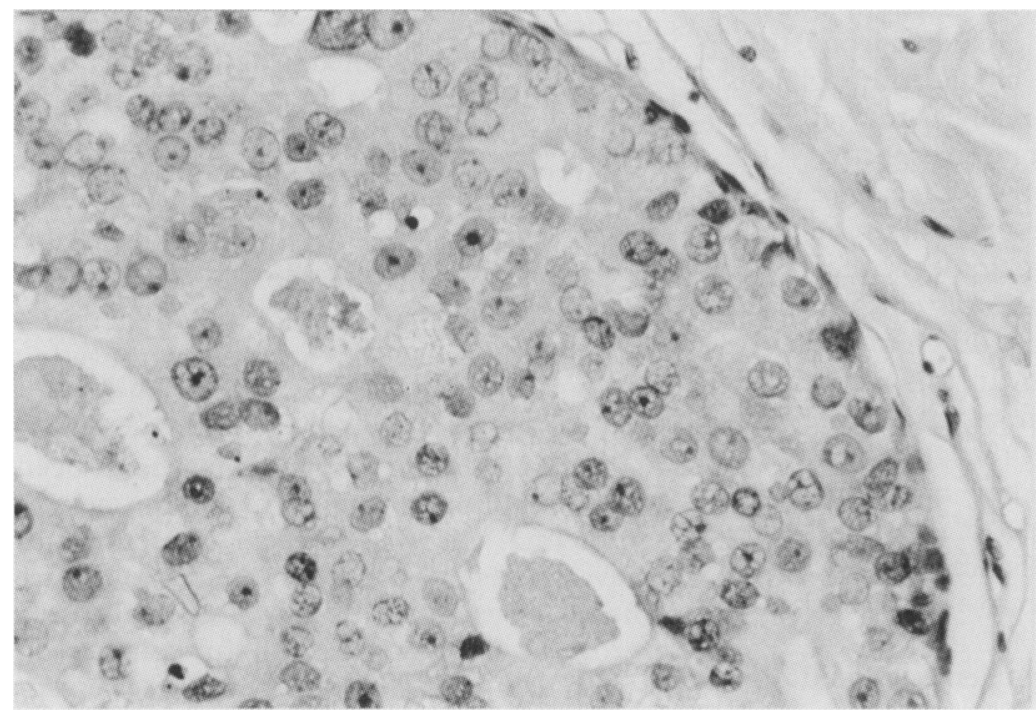

Figure 2 Intermediately differentiated DCIS showing a cribriform growth pattern. There is some nuclear pleomorphism but this is less marked than that seen in poorly differentiated DCIS. Cell polarisation is evident focally around intercellular spaces.

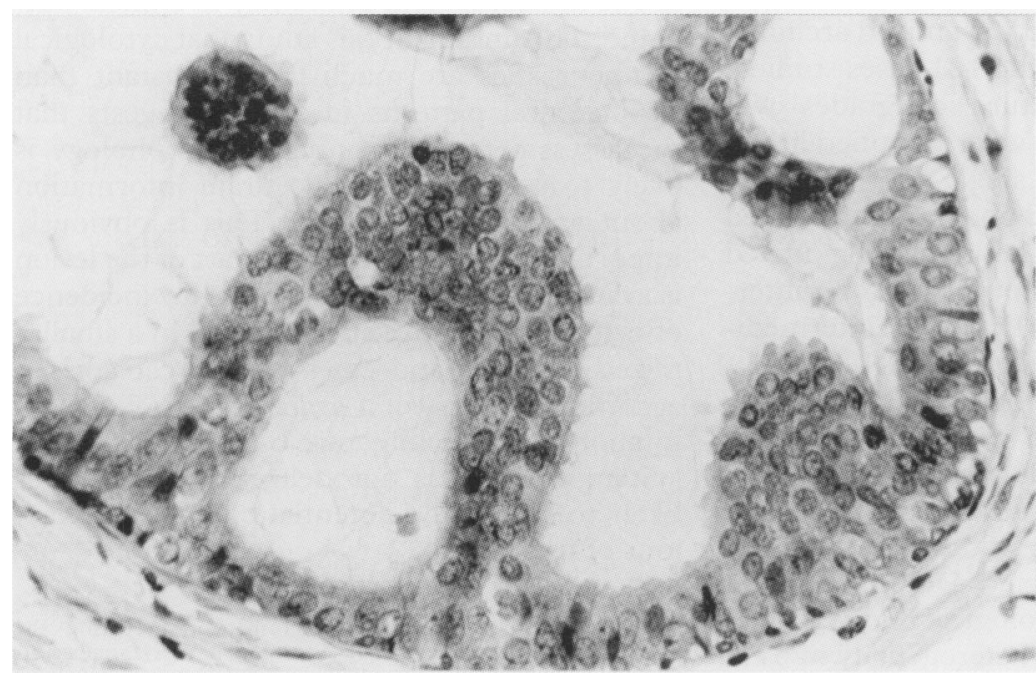

Figure 3 Well differentiated DCIS showing micropapillary and cribriform growth patterns. The nuclei are uniform and there is good polarisation with orientation of the cells towards the duct lumen. of Holland et $a l .{ }^{15}$ This system categorises DCIS into three groups based on nuclear morphology and, to a lesser extent, on cell polarisation. Poorly differentiated DCIS (fig 1) comprises cells containing large, pleomorphic nuclei with no tendency to cell polarisation. Central necrosis is a frequent but not invariable finding. In intermediately differentiated DCIS (fig 2) the nuclei are less pleomorphic and cell polarisation is present focally. Well differentiated DCIS (fig 3) comprises small cells with monomorphic nuclei and prominent polarisation of cells. Cases were described as pure when only one grade was present and as mixed when more than one grade was observed.

Architecturally DCIS was classified as solid, cribriform, micropapillary, or papillary. Because of the lack of uniform agreement as to what constitutes comedo, ${ }^{8}{ }^{18}$ we did not use this as an architectural category. Cases in which the cells surrounding an area of central necrosis showed a solid growth pattern were classified as solid. Cases were described as pure when only one architectural pattern was present and as mixed when more than one pattern was seen. Necrosis was assessed as an independent parameter and was scored as absent, focal, or extensive.

Each case was graded by two observers and any discrepancies resolved by consultation at the double-headed microscope. The incidence of cytological heterogeneity was compared with that of architectural heterogeneity. The relation between the presence of necrosis and DCIS grade was also evaluated.

\section{Results}

Cytological heterogeneity was much less common than architectural heterogeneity in individual DCIS lesions (fig 4). One hundred and two cases $(84 \%)$ showed a single DCIS grade; 65 poorly differentiated, 25 intermediately differentiated, and 12 well differentiated. The remaining 19 cases (16\%) showed mixed grades, 16 of which included areas of poorly differentiated DCIS. In contrast a pure architectural pattern was observed in only 46 cases $(38 \%)$; 38 solid, 5 cribriform, 2 micropapillary, and 1 papillary. Mixed architectural patterns were identified in the remaining 75 cases $(62 \%)$; two patterns in 60 and three patterns in 15. Necrosis was present in 99 cases (82\%) and was extensive in $61(50 \%)$. Extensive necrosis was documented in 28 of 46 cases with a pure architecture, in 33 of 75 with a mixed architecture, and was observed in association with solid, cribriform, and micropapillary growth patterns. Fifty eight of the 61 lesions showing extensive necrosis were graded as poorly differentiated and three as intermediately differentiated. The presence of extensive necrosis was significantly related to DCIS grade $\left(\chi^{2}=32.14, \mathrm{p}<0.0001\right)$.

\section{Discussion}

The cytology based classification system used in this study was developed by a group of European pathologists in response to the need for a clinically relevant method for classifying DCIS. ${ }^{15}$ This system has been validated by 


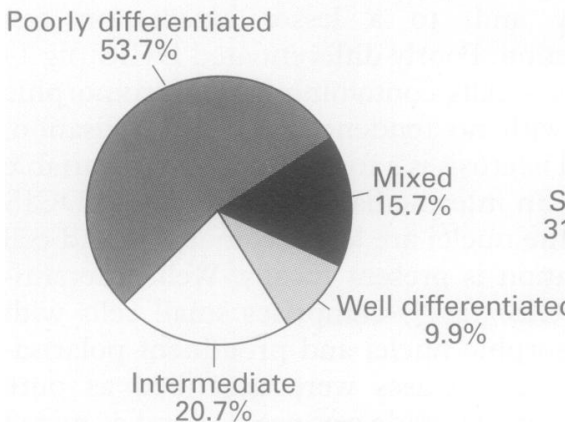

Figure 4 DCIS classified according to a cytology based system $(A)$ and the traditional architecture based system (B). The percentage of cases showing mixed architectural patterns is much greater than that showing a mixture of cytological grades.

comparison with oncogene and cell proliferation markers, which have been shown to be related to prognosis in invasive breast carcinoma.${ }^{19}$ In addition a close correlation has been demonstrated between DCIS differentiation and the histological grade of the invasive component in tumours composed of in situ and invasive ductal carcinoma. ${ }^{20}$ This suggests that the grade of the invasive carcinoma developing from DCIS can be predicted from study of the DCIS component alone. Although this DCIS classification system has not yet been evaluated prospectively or retrospectively the results of these correlative studies suggest that the system is likely to be clinically predictive. Morphologically, nuclear grade is the major diagnostic criterion but the system also takes account of architectural differentiation by assessing cell polarisation. In this respect this classification system is more in keeping with the modern approach to the classification of invasive lesions than other cytology based DCIS classification systems. It also differs from other cytology based classification systems in not relying on comedo necrosis as a diagnostic criterion.

In this study we have demonstrated that lesional heterogeneity is much less common when DCIS is classified using a cytology based classification system instead of the traditional architectural approach. Mixed grade was documented in $16 \%$ compared with mixed architectural patterns in $62 \%$ of the 121 cases studied. In cases where a mixture of grades was observed only two grades were identified in any one case. Apart from a single case in which separate areas of well and poorly differentiated DCIS were identified, the remaining mixed cases showed a combination of well and intermediately differentiated DCIS or intermediately and poorly differentiated DCIS.

Architectural heterogeneity in DCIS has been assessed by others with reported incidences of up to $61 \%$ as in our study. ${ }^{111421}$ Using nuclear grade and the traditional architecture based system in a study of 112 cases of mammographically detected DCIS, Harrison et al recently reported cytological heterogeneity in $16 \%$ and architectural heterogeneity in $61 \%$ of cases, almost identical results to those detailed in this study. ${ }^{21}$ They also reported a similar incidence of necrosis, the presence of which was closely associated with high grade DCIS. This similarity of results reinforces the view that cytological characteristics are more constant than architectural patterns in DCIS. It also suggests that DCIS classification systems that rely primarily on the evaluation of nuclear morphology are reproducible in different centres.

DCIS lesions featuring prominent luminal necrosis were traditionally designated comedo, and a broad subdivision of DCIS into comedo and non-comedo categories has been proposed. There is, however, little agreement about what precisely constitutes comedo. Definitions include the presence of extensive necrosis, central necrosis surrounded by a proliferation of cells showing a solid growth pattern, and central necrosis in association with high nuclear grade. This study has demonstrated a significant correlation between the presence of extensive necrosis and poor DCIS differentiation. This is in keeping with the results of other studies reporting a strong correlation between the presence of necrosis and high nuclear grade, ${ }^{18}$ and the development of invasive carcinoma following previous excision of DCIS. ${ }^{1822}$ However, in our study, necrosis was not identified in all cases classified as poorly differentiated by cytological criteria, supporting the views of others that the categorisation of DCIS by necrosis only is over simplistic.

The natural history of DCIS is still unfolding. The introduction of mammography screening means that many of these lesions are now being detected and surgically removed at an early stage in their evolution. Histopathologists are increasingly diagnosing low grade DCIS that in the past may not have been recognised. Similarly, heightened awareness of the potential of DCIS to recur, sometimes as an invasive lesion, has led surgeons to ensure complete excision with a rim of normal tissue. In order to make any meaningful comparison between data from different centres it is essential that pathologists employ a classification system that is reproducible and generates defined categories that can be related to prognostic indicators and ultimately to clinical outcome. The finding in this study that cytological characteristics are much more constant than architectural patterns in DCIS suggests that the assessment of cytonuclear morphology is likely to generate more accurate information about an individual lesion. This is obviously important in cases where only part of the lesion is available for examination. The low incidence of cytological heterogeneity results in a smaller mixed category and cases can be categorised according to the worst grade. This is less easy to apply to an architecture based classification system where it is not clear which pattern is likely to dictate the potential biological behaviour of the lesion.

We are grateful to $\mathrm{Mr}$ Steven Toms for preparing the photographs and to Mrs Jacquie Fearnley for secretarial assistance.

1 Lagios MD, Margolin FR, Westdahl PR, Rose ME Mammographically detected duct carcinoma in situ. 
Frequency of local recurrence following tylectomy and prognostic effect of nuclear grade on local recurrence. Cancer 1989;63:618-24.

2 Lagios MD. Duct carcinoma in situ. Pathology and treatment. Surg Clin North Am 1990;70:853-71.

3 Locker AP, Horrocks C, Gilmour AS, Ellis IO, Dowle CS, Elston CW, et al. Flow cytometric and histologic analysis of ductal carcinoma in situ of the breast. $\mathrm{Br} \mathcal{F}$ Surg 1990;77:564-7.

4 Killeen JL, Namiki H. DNA analysis of ductal carcinoma in situ of the breast. A comparison with histological features. Situ of the 1991;68:2602-7.

5 Barnes DM, Meyer JS, Gonzalez JG, Gullick WJ, Millis RR. Relationship between c-erbB-2 immunoreactivity and thymidine labelling index in breast carcinoma in situ. Breas Cancer Res Treat 1991;18:11-17.

6 van Dongen JA, Holland R, Peterse JL, Fentiman IS, Lagios MD, Millis RR, et al. Ductal carcinoma in situ of the breast second EORTC consensus meeting. Eur 7 Cancer 1992;28: 626-9.

7 Bur ME, Zimarowski MJ, Schnitt SJ, Baker S, Lew R. Estrogen receptor immunohistochemistry in carcinoma in Estrogen receptor immunohistochemistry
situ of the breast. Cancer 1992;69:1174-81.

8 Bellamy COC, McDonald C, Salter DM, Chetty U, AnderBellamy COE, McDonald C, Salter DM, Chetty U, Anderevance of histologic categorization. Hum Pathol 1993;24 evance

9 Solin LJ, Yeh I-T, Kurtz J, et al. Ductal carcinoma in situ (intraductal carcinoma) of the breast treated with breastconserving surgery and definitive irradiation. Correlation of pathologic parameters with outcome of treatment. Cancer 1993;71:2532-42.

10 Poller DN, Roberts EC, Bell JA, Elston CW, Blamey RW, Ellis IO. p53 protein expression in mammary ductal carcinoma in situ: relationship to immunohistochemical expression of estrogen receptor and c-erbB-2 protein. Hum Pathol 1993;24:463-8.

11 Leal CB, Schmitt FC, Bento MJ, Maia NC, Lopes CS Ductal carcinoma in situ of the breast. Histologic categorization and its relationship to ploidy, and immunohistochemical expression of hormone receptors, p53, and c-erbB-2 protein. Cancer 1995;75:2123-31.
12 Azzopardi JG. Epitheliosis and in situ carcinoma. In: Bennington JL, ed. Problems in breast pathology. Philadelphia: Saunders, 1979:128-46.

13 Rogers LW. Carcinoma in situ (CIS). In: Page DL Anderson TJ, eds. Diagnostic histopathology of the breast. Edinburgh: Churchill Livingstone, 1987:157-92.

14 Lennington WJ, Jensen RA, Dalton LW, Page DL. Ductal carcinoma in situ of the breast. Heterogeneity of individual carcinoma in situ of the breast.

15 Holland R, Peterse JL, Millis RR, Eusebi V, Faverly D, van de Vijver MJ, et al. Ductal carcinoma in situ: a proposal fo a new classification. Semin Diagn Path 1994;11:167-80.

16 Poller DN, Silverstein MJ, Galea M, et al. Ductal carcinoma in situ of the breast: a proposal for a new simplified histological classification. Association between cellular proliferation and c-erbB-2 protein expression. Mod Pathol 1994; $7: 257-62$.

17 Silverstein MJ, Poller DN, Waisman JR, Colburn WJ, Barth A, Gierson ED, et al. Prognostic classification of breast ductal carcinoma in situ. Lancet 1995;345:1154-7.

18 Bobrow LG, Happerfield LC, Gregory WM, Millis RR Ductal carcinoma in situ: assessment of necrosis and nuclear morphology and their association with biological markers. F Pathol 1995;176:333-41.

19 Bobrow LG, Happerfield LC, Gregory WM, Springall RD, Millis RR. The classification of ductal carcinoma in situ and its association with biological markers. Semin Diagn and its association with

20 Lampejo OT, Barnes DM, Smith P, Millis RR. Evaluation of infiltrating ductal carcinomas with a DCIS component: correlation of the histologic type of the in situ componen with grade of the infiltrating component. Semin Diagn Pathol 1994;11:215-22.

21 Harrison M, Coyne JD, Gorey T, Dervan PA. Comparison of cytomorphological and architectural heterogeneity in mammographically detected ductal carcinoma in situ. Histopathology 1996;28:445-50.

22 Ottesen GL Graversen HP, Blichert-Toft M, Zedeler K, Andersen JA. Ductal carcinoma of the female breast. Short term results of a prospective nationwide study. Am $\mathcal{F}$ Surg Pathol 1992;16:1183-96. 